\title{
ARTICLE
}

\section{1-year outcomes of the Xen45 glaucoma implant}

\author{
Michael Smith $\mathbb{1}^{1} \cdot$ Roselin Charles ${ }^{1} \cdot$ Ahmed Abdel-Hay $\mathbb{1}^{2} \cdot$ Brinda Shah $^{3} \cdot$ Daniel Byles $^{1} \cdot$ Lei-Ai Lim $^{2} \cdot$ \\ Jonathan Rossiter ${ }^{2} \cdot$ Chih-Hung Kuo ${ }^{4,5} \cdot$ Peter Chapman $^{3} \cdot$ Shalini Robertson $^{3}$
}

Received: 24 May 2018 / Revised: 18 September 2018 / Accepted: 23 September 2018 / Published online: 14 December 2018

(c) The Royal College of Ophthalmologists 2018

\begin{abstract}
Objectives To describe the 12-month outcomes of the Xen45 glaucoma stent.

Methods Non-comparative retrospective study of all cases who underwent Xen glaucoma surgery in April 2017 or earlier and completed 12 months of follow-up. The primary outcome measures were intraocular pressure (IOP) reduction and number of glaucoma medications at 12 months postoperatively. The secondary outcome measures were surgical complications and the success rate of surgery at 1 year. Success rate was defined according to the multiple IOP thresholds of $15 \mathrm{mmHg}, 18 \mathrm{mmHg}$, and $21 \mathrm{mmHg}$ with all requiring a drop of $20 \%$ and no additional glaucoma surgery. Revision or needling of the Xen conjunctival bleb was not considered to constitute a surgical failure.

Results Sixty-eight eyes were included in the study. Mean IOP dropped from $22.1 \mathrm{mmHg}$ preoperatively to $14.8 \mathrm{mmHg}$ at 12 months, a $33 \%$ drop $(p<0.0001)$. Mean number of glaucoma medications reduced from 2.9 preoperatively to 1.1 at 12 months $(p<0.0001)$. In total, $54.4 \%$ of cases were back on glaucoma medications by 12 months. Success rate varied from $32.4 \%$ when defined as IOP $\leq 15 \mathrm{mmHg}$ and $\geq 6 \mathrm{mmHg}$ and $\geq 20 \%$ reduction without medications to $70.6 \%$ when defined as IOP $\leq 21 \mathrm{mmHg}$ and $\geq 6 \mathrm{mmHg}$ and $\geq 20 \%$ reduction with or without medications. Thirty cases (44.1\%) required bleb needling or surgical revision.

Conclusions The Xen45 is effective at reducing IOP and glaucoma medication use at 12 months postoperatively. Patients considering this procedure should be warned that by 12 months postoperatively there is a significant chance of requiring postoperative bleb intervention and glaucoma drops.
\end{abstract}

\section{Introduction}

Over the last decade, the number of laser and surgical treatment options available for glaucoma patients has increased. In addition to longstanding treatments such as trabeculectomy and laser trabeculoplasty, glaucoma specialists have reduced their threshold for glaucoma drainage devices and in recent years the term Minimally Invasive

Michael Smith

Michael.smith26@nhs.net

1 West of England Eye Unit, Royal Devon and Exeter Hospital, Exeter, UK

2 Ophthalmology Department, Musgrove Park Hospital, Taunton, UK

3 Ophthalmology Department, Yeovil Hospital, Yeovil, UK

4 Ophthalmology Department, Gold Coast University Hospital, Gold Coast, Australia

5 Sydney Medical School, Sydney, Australia
Glaucoma Surgery (MIGS) has been introduced to cover several novel surgical devices $[1,2]$. These devices are typically inserted via an ab interno approach and involve minimal or no conjunctival manipulation [3]. Most MIGS devices work by improving outflow through the physiological pathways of Schlemms Canal or the supraciliary space rather than the non-physiological subconjunctival route of traditional glaucoma drainage surgery. This is likely to explain why initial studies suggest they are less efficacious than these procedures but with a lower rate of hypotony related complications. The XEN implant (Allergan, Dublin, Ireland) and the Innfocus Microshunt (Santen Pharm Ltd, Osaka, Japan), result in subconjunctival drainage and therefore offer the possibility of achieving intraocular pressure (IOP) similar to trabeculectomy but, as they have less scleral and conjunctival manipulation, with a more rapid recovery time and less complications [4]. The evidence for these procedures, however, remains limited.

The Xen implant, which is the subject of this paper, was initially designed with an internal diameter of $140 \mu \mathrm{m}$ or 63 $\mu \mathrm{m}$ and several reports described encouraging results $[5,6]$. 
The Xen45, which has an internal diameter of $45 \mu \mathrm{m}$, is now the only commercially available device and has FDA approval. Published evidence for this device is limited, often describing the single surgeon results of early adopters of this procedure [7-13]. As a result, many glaucoma specialists remain unclear on what benefits and risks Xen surgery offers to their patients. In a survey of glaucoma specialists in the United Kingdom and Ireland published in 2017 , less than one in five were undertaking this procedure [14].

This paper presents the 12-month results of our initial cohort of patients who underwent glaucoma surgery with the Xen45. We hope our experience will provide valuable information to glaucoma patients and their clinicians when considering this procedure.

\section{Methods}

This study includes data collected at our three institutions. As it is a retrospective case note review, formal ethics approval was not needed, but local clinical governance approval for data collection was obtained.

Since commencing Xen glaucoma surgery to our patients we have been routinely collecting outcome data on all cases. In April 2018, we identified all cases at our institutions who have completed 12 months of follow-up.

The inclusion criterion was therefore all cases who underwent Xen glaucoma surgery in April 2017 or earlier. The exclusion criterion was failure to complete 12 months of follow-up.

The primary outcome measures were IOP reduction and number of glaucoma medications at 12 months postoperatively. The secondary outcome measures were complications owing to the surgery and the success rate of surgery at 1 year. In order to allow comparison with previous reports we defined success as below [7-13].

1. IOP $\leq 21 \mathrm{mmHg}$ and $\geq 6 \mathrm{mmHg}$ and $\geq 20 \%$ reduction from baseline with no additional glaucoma procedures.

2. $\mathrm{IOP} \leq 18 \mathrm{mmHg}$ and $\geq 6 \mathrm{mmHg}$ and $\geq 20 \%$ reduction from baseline with no additional glaucoma procedures.

3. IOP $\leq 15 \mathrm{mmHg}$ and $\geq 6 \mathrm{mmHg}$ and $\geq 20 \%$ reduction from baseline with no additional glaucoma procedures.

Complete success was defined as achieving the outcomes above without the need for glaucoma medications, whereas qualified success was achieving the outcomes with or without medications. Revision or needling of the Xen conjunctival bleb was recorded but not considered to constitute a surgical failure.

\section{Surgical technique}

All cases were performed by one of five glaucoma subspecialists, all with extensive experience of traditional glaucoma drainage surgery.

In total, $0.2 \mathrm{mls}$ of $0.02 \%$ Mitomycin-C was injected into the subconjunctival/subtenon space in the supranasal quadrant at the start of surgery (unlicensed indication). A clear corneal incision was made in the inferotemporal quadrant and the anterior chamber filled with viscoelastic. Using the pre-loaded injector the Xen45 device was implanted via an ab interno approach in the supranasal quadrant. The viscoelastic was then removed and intracameral antibiotics given. If combined with cataract surgery, the Xen implantation was performed following insertion of the intraocular lens.

Postoperatively, all glaucoma medications were stopped and antibiotic drops were used for 1 week. Steroid drops were used for at least 4 weeks then tapered according to the degree of inflammation present.

\section{Statistical analysis}

Data were entered into Microsoft Excel (Microsoft Corporation) and statistical analysis performed using Graphpad Prism Version 7.0c (GraphPad Software Inc). Shapiro-Wilk tests were used to test for presence or absence of normal distribution in the data. Comparison between preoperative and postoperative data were performed by using the Wilcoxon test, with a $P$ value of 0.05 or less considered statistically significant. If the patient underwent further laser or surgery for glaucoma in the same eye data were censored at the date of the additional procedure.

\section{Results}

By April 2017, we had undertaken 75 procedures, meaning 12-month data should have been available when we undertook the data analysis in April 2018. Seven patients were excluded as 12-month data were not available (six lost to follow-up and one patient died). Sixty-eight eyes of 68 patients were therefore included in the study. The preoperative characteristics are summarized in Table 1.

Ten cases $(14.7 \%)$ underwent Xen glaucoma implant surgery combined with phacoemulsification cataract surgery with 58 cases $(85.3 \%)$ Xen alone.

Table 2 summarizes the study outcomes. Mean IOP dropped from $22.1 \mathrm{mmHg}$ preoperatively to $14.8 \mathrm{mmHg}$ at 12 months, a drop of $33 \%$. The mean number of glaucoma medications reduced from 2.9 preoperatively to 1.1 at 12 months, with $54.4 \%$ of cases back on glaucoma medications by 12 months. The mean (and 95\% confidence 
intervals) IOP over the first 12 months are represented graphically in Fig. 1.

The success rate of surgery as defined by a range of target IOPs is summarized in Table 3. Of the 20 cases $(29.4 \%)$ that did not meet the least strict success criteria (IOP $\leq 21 \mathrm{mmHg}$ and $\geq 6 \mathrm{mmHg}$ and $\geq 20 \%$ reduction from baseline with or without medications with no additional glaucoma procedures except Xen needling or revision) 2 ( $2.9 \%$ of total) had undergone further surgery in the form of trabeculectomy, whereas $18(26.5 \%)$ did not achieve an adequate IOP reduction. There were no cases of failure owing to hypotony at 12 months.

Revision or needling of the Xen conjunctival bleb was recorded but not considered to constitute a surgical failure. Twenty-six cases $(38.2 \%)$ underwent bleb needling (all with Mitomycin-C), with one case undergoing two procedures and three cases three procedures. Seven cases underwent open surgical revision (10.3\%). In three cases $(4.4 \%)$, this was owing to symptomatic overhanging blebs and in one

Table 1 Preoperative characteristics

\begin{tabular}{lll}
\hline & Mean (median, SD) & Range \\
\hline Sex & $33(47.5 \%)$ female \\
& $35(51.5 \%)$ male \\
Age & $76(78,10)$ years & 48-97 Years \\
Intraocular pressure & $12.1(20,6.4) \mathrm{mmHg}$ & $10-44 \mathrm{mmHg}$ \\
Glaucoma medications & $2.85(3,0.8)$ & $1-4$ \\
Mean deviation & $-11.07(-9.79,7.78)$ & 0.58 to -32.00 \\
Previous glaucoma & Laser trabeculoplasty $44(64.7 \%)$ \\
procedures & Endocyclophotocoagulation $4(5.9 \%)$ \\
& Laser peripheral iridotomy 4 (5.9\%) \\
& Trabeculectomy 3 (4.4\%) \\
& Trabectome 1 (1.5\%) \\
& No previous laser or surgery 21 (30.8\%) \\
& Phakic 23 (33.8\%) \\
& Pseudophakic 45 (66.2\%) \\
Lens status & Primary open angle/normal tension \\
& glaucoma 59 (86.7\%) \\
Diagnosis & Pseudoexfoliative glaucoma 4 (5.9\%) \\
& Primary angle closure glaucoma 3 \\
& (4.4\%) \\
& Ocular hypertension 2 (2.9\%) \\
&
\end{tabular}

$S D$ standard deviation case $(1.5 \%)$ owing to tube exposure. The remaining three cases $(4.4 \%)$ underwent revision owing to inadequate IOP control. In two of these cases, the revision was following a failed bleb needling procedure. In total 30 cases $(44.1 \%)$ required bleb needling with $\mathrm{MMC}$ or surgical revision. The success rate at 12 months of the group which underwent bleb needling or revision was similar to the group which did not undergo further intervention. In total, $70 \%$ of this group met the least strict success criteria (IOP $\leq 21 \mathrm{mmHg}$ and $\geq 6$ $\mathrm{mmHg}$ and $\geq 20 \%$ reduction from baseline with or without drops) compared with $71 \%$ of the group that did not undergo further intervention. In addition, $33 \%$ met the strictest success criteria definition (IOP $\leq 15 \mathrm{mmHg}$ and $\geq 6$ $\mathrm{mmHg}$ and $\geq 20 \%$ reduction from baseline with no drops) compared with $32 \%$ of the group which did not undergo bleb needling or revision.

Fifeteen cases $(22.1 \%)$ developed a significant perioperative or postoperative complication. At the time of surgery, six cases (10.3\%) developed a hyphaema, whereas one case $(1.5 \%)$ suffered iris prolapse. In the early postoperative period, five cases $(7.4 \%)$ developed a period of hypotony and one case $(1.5 \%)$ choroidal effusions. With the exception of one case all of these resolved without further intervention, with the final case undergoing revision surgery. In the late postoperative period, one (1.5\%) case underwent successful revision for tube exposure. A final case developed hypotony and severe choroidal effusions following tube revision and required further revision with pericardial patch graft. Three cases $(4.4 \%)$ required bleb revision for symptomatic overhanging blebs.

\section{Discussion}

Faced with a patient with uncontrolled glaucoma, the glaucoma specialist has an ever-increasing number of laser and surgical options to call upon. At present, the decision on which procedure represents the best balance of risks and benefits for the individual patient is made difficult by the limited availability of outcome data, especially for the newer glaucoma procedures such as the Xen gel stent. The Xen45 has FDA approval although the United Kingdom National Institute for Clinical Excellence recommends that owing to the limited evidence base for the device the outcomes of all cases should be audited [15].
Table 2 Statistical comparison of outcome measures

\begin{tabular}{llll}
\hline & $\begin{array}{l}\text { Preoperative }(n=68) \\
\text { Mean (median, SD) }\end{array}$ & $\begin{array}{l}\text { 12 Months }(n=68) \\
\text { Mean (median, SD) }\end{array}$ & $P$ value for difference \\
\hline Intraocular pressure $(\mathrm{mmHg})$ & $22.1(20,6.4)$ & $14.8(13.5,5.1)$ & $<0.0001$ \\
Glaucoma medications & $2.9(3,0.8)$ & $1.1(1,1.1)$ & $<0.0001$ \\
\hline
\end{tabular}

$S D$ standard deviation 
In this series, which represents the initial cases performed in three units in the South West of England, the mean IOP dropped from $22.1 \mathrm{mmHg}$ preoperatively to 14.8 $\mathrm{mmHg}$ at 12 months, a drop of $33 \%$. The mean number of glaucoma medications reduced from 2.9 preoperatively to 1.1 at 12 months, with $54.4 \%$ of cases back on glaucoma medications by 12 months. In all, $44.1 \%$ required bleb needling or revision during the 12 -month period, with $2.9 \%$ going on to trabeculectomy during this time period.

Despite the lack of randomized control trial data, several non-comparative studies have reported 12-month outcome data, and these are summarized in Table 4, alongside the results of this paper for comparison. Although the results are not entirely consistent between studies, the results broadly agree with the outcomes in our series. It appears that at

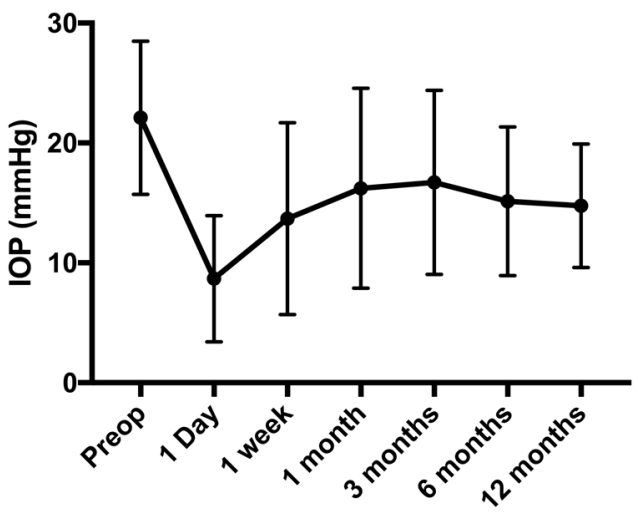

Time

Fig. 1 Mean IOP (mmHg) over time with 95\% confidence intervals
12 months, Xen45 achieves an IOP in the mid-teens, with a substantial drop in medications. The reported needling/ revision rate is variable, and although the reasons for this are not clear it may relate to a number of variables including patient selection and individual surgeons' thresholds for intervention. Based on the published results, however, it appears that $25-50 \%$ of patients require bleb intervention in the first year postoperatively following Xen surgery. This compares to a $14 \%$ needling rate in the trabeculectomy arm of the Primary Tube Versus Trabeculectomy Study and it does appear that despite the lesser degree of conjunctival manipulation during Xen surgery compared with trabeculectomy postoperative loss of IOP control owing to subconjunctival fibrosis is more common [16].

The use of Mitomycin-C and the subconjunctival drainage route of Xen surgery means it was never likely to achieve the safety profile of the MIGS devices, which work by improving outflow through the physiological pathways of Schlemms Canal or the supraciliary space, and $22.1 \%$ of patients in our series suffered a significant complication.

The limitations of this study mainly relate to its retrospective nature. In addition, this case series represents the first Xen cases we have undertaken and therefore may include a learning curve effect, although as experienced glaucoma surgeons we did not find the procedure technically challenging. Furthermore, as can be seen from Table 1 our cases represent a heterogenous group, which can make it difficult to extrapolate our results to specific situations. As the evidence base for the Xen glaucoma implant increases we hope it will become clearer which subgroups of glaucoma patients are best served by this procedure.
Table 3 Success rate of Xen surgery

\begin{tabular}{lll}
\hline Definition of success & Complete success & Qualified success \\
\hline IOP $\leq 21 \mathrm{mmHg}$ and $\geq 6 \mathrm{mmHg}$ and $\geq 20 \%$ reduction from baseline & $24(35.3 \%)$ & $48(70.6 \%)$ \\
IOP $\leq 18 \mathrm{mmHg}$ and $\geq 6 \mathrm{mmHg}$ and $\geq 20 \%$ reduction from baseline & $23(33.8 \%)$ & $46(67.6 \%)$ \\
IOP $\leq 15 \mathrm{mmHg}$ and $\geq 6 \mathrm{mmHg} \geq 20 \%$ reduction from baseline* & $22(32.4 \%)$ & $42(61.8 \%)$ \\
\hline
\end{tabular}

*Any additional surgery or laser for intraocular pressure were considered surgical failures but revision or needling of the Xen conjunctival bleb was permitted

Table 4 Published reports of the Xen45 and comparison with this study

\begin{tabular}{|c|c|c|c|c|c|c|}
\hline \multirow[t]{2}{*}{ Author } & \multirow[t]{2}{*}{ Study details } & \multicolumn{2}{|c|}{ Preoperative } & \multicolumn{2}{|c|}{12 months } & \multirow[t]{2}{*}{ Bleb needling or revision $(\%)$} \\
\hline & & IOP & Medications & IOP & Medications & \\
\hline Galal [6] & 13 eyes (10 with phaco) & 16 & 1.9 & 12 & 0.3 & $30.7 \%$ \\
\hline Perez-Torregrosa [7] & 30 eyes (all with phaco) & 21.2 & 3.07 & 15.03 & 0.17 & $23.3 \%$ \\
\hline De Gregoria [8] & 41 eyes (all with phaco) & 22.5 & 2.5 & 13.1 & 0.4 & $4.8 \%$ \\
\hline Tan [9] & 39 eyes (no phaco) & 24.9 & 3.0 & 14.5 & 0.7 & $51.3 \%$ \\
\hline Hengerer [10] & 148 eyes (unclear $\%$ with phaco) & 32.2 & 3.13 & 14.24 & 0.3 & $27.7 \%$ \\
\hline Fea [11] & 12 eyes ( 2 with phaco) & 21.8 & 2.92 & 14.9 & 0.5 & $50 \%$ \\
\hline Grover [12] & 65 eyes (no phaco) & 25.1 & 3.5 & 15.9 & 1.7 & $32.3 \%$ \\
\hline This study & 68 eyes ( $14.7 \%$ with phaco) & 22.1 & 2.9 & 14.8 & 1.1 & $44.1 \%$ \\
\hline
\end{tabular}


An additional weakness of our study is that in a proportion of the cases $(14.7 \%)$ the Xen surgery was combined with phacoemulsification and in these cases we are not able to separate the IOP-lowering effect of the cataract surgery from the IOP lowering effect of the Xen. If we excluded the combined phaco-xen cases from our statistical analysis, however, we did not find an appreciable difference in the results, with the Xen only cases IOP dropping from 21.9 $\mathrm{mmHg}$ to $15.2 \mathrm{mmHg}$, a drop of $30.5 \%$. The success rate was also similar if we excluded the phaco-Xen cases, with 33 and $71 \%$ of cases meeting the most strict (IOP $\leq 15$ $\mathrm{mmHg}$ and $\geq 6 \mathrm{mmHg}$ and $\geq 20 \%$ reduction from baseline with no drops) and least strict (IOP $\leq 21 \mathrm{mmHg}$ and $\geq 6$ $\mathrm{mmHg}$ and $\geq 20 \%$ reduction from baseline with or without drops), respectively. In addition a recent review and metaanalysis examining the effect of phaco alone on IOP in glaucoma patients concluded that at 1 year post phacoemulsification the mean percentage drop in IOP in glaucoma patients was only $14.4 \%$ [17]. Further research is needed to the relative effectiveness of phaco-Xen and Xen without phaco.

In conclusion, the Xen45 is effective at reducing IOP and glaucoma medication use at 12 months postoperatively. Patients considering this procedure should be warned that by 12 months postoperatively, there is a significant chance of requiring postoperative bleb intervention and glaucoma drops.

Further research is needed to determine the place of Xen45 in the ever-increasing number of procedures available to glaucoma patients. Until then we would recommend advising patients on the best option for them, balancing the relative efficacy and side effect profile of each procedure against the outcome required for each individual patient.

\section{Study Highlights}

\section{What was known before}

- The number of surgical options to treat glaucoma has increased in recent years. The Xen45 implant works via subconjunctival drainage and therefore offers the possibility of achieving IOPs similar to trabeculectomy but with less side effects. Evidence for the safety and efficacy of the Xen45 is limited.

\section{What this study adds}

- The Xen45 is effective at reducing IOP and glaucoma medication use at 12 months postoperatively. Patients considering this procedure should be warned that by
12 months postoperatively there is a significant chance of requiring postoperative bleb intervention and glaucoma drops to achieve adequate IOP control.

\section{Compliance with ethical standards}

Conflict of interest All authors have received meeting sponsorship from Allergan Ophthalmology except CK. MS has received research funding from Allergan.

Publisher's note: Springer Nature remains neutral with regard to jurisdictional claims in published maps and institutional affiliations.

\section{References}

1. Vinod K, Gedde SJ, Feuer WJ, et al. Practice preferences for glaucoma surgery: a survey of the American glaucoma Society. J Glaucoma. 2017;26:687-93.

2. Richter GM, Coleman AM. Minimally invasive glaucoma surgery: current status and future prospects. Clin Ophthalmol. 2016;10:189-206.

3. Lavia C, Dallorto L, Maule M, Ceccarelli M, Fea AM. Minimallyinvasive glaucoma surgeries (MIGS) for open angle glaucoma: a systematic review and meta-analysis. PLoS ONE. 2017;12: $\mathrm{e} 0183142$.

4. Green W, Lind J, Sheybani A. Review of the Xen Gel Stent and InnFocus MicroShunt. Curr Opin Ophthalmol. 2018;29: 162-70.

5. Sheybani A, Lenzhofer M, Hohensinn M, et al. Phacoemulsification combined with a new ab interno gel stent to treat openangle glaucoma: pilot study. J Cataract Refract Surg. 2015;41:1905-9.

6. Sheybani A, Dick B, Ahmed IIK. Early clinical results of a novel ab interno gel stent for the surgical treatment of open-angle glaucoma. J Glaucoma. 2016;25:e691-e696.

7. Galal A, Bilgic A, Eltanamly R, et al. XEN glaucoma implant with mitomycin C 1-year follow-up: result and complications. J Ophthalmol. 2017:5457246.

8. Pérez-Torregrosa VT, Olate-Pérez Á, Cerdà-Ibáñez M, et al. Combined phacoemulsification and Xen45 surgery from temporal approach and 2 incisions. Arch Soc Esp Oftalmol. 2016;91: 415-21.

9. De Gregoria A, Pedrotti E, Russo L, et al. Minimally invasive combined glaucoma and cataract surgery: clinical results of the smallest ab interno gel stent. Int Ophthalmol. 2018;38: $1129-1134$.

10. Tan SZ, Walkden A, Au L. One-year result of XEN45 implant for glaucoma: efficacy, safety, and postoperative management. Eye. 2018;32:324-32.

11. Hengerer FH, Kohnen T, Mueller M, Conrad-Hengerer I. Ab interno gel implant for the treatment of glaucoma patients with or without prior glaucoma surgery: 1-year results. J Glaucoma. 2017;26:1130-6.

12. Fea AM, Spinetta R, Cannizzo PML, et al. Evaluation of bleb morphology and reduction in IOP and glaucoma medication following implantation of a novel gel stent. J Ophthalmol. 2017:9364910.

13. Grover DS, Flynn WJ, Bashford KP, et al. Performance and safety of a new ab interno gelatin stent in refractory glaucoma at 12 months. Am J Ophthalmol. 2017;183:25-36. 
14. Rodriguez-Una I, Azuara-Blanco A, King AJ. Survey of glaucoma surgical preferences and post-operative care in the United Kingdom. Clin Exp Ophthalmol. 2017;45:232-40.

15. National Institute for Health and Clinical Excellence. Microinvasive subconjunctival insertion of a trans-scleral gelatin stent for primary open-angle glaucoma 2018. Available from: https://www.nice.org.uk/guidance/ipg612/chapter/1-Recommenda tions.
16. Gedde SJ, Feuer WJ, Shi W, et al. Treatment outcomes in the primary tube versus trabeculectomy study after 1 year of followup. Ophthalmology. 2018;125:650-63.

17. Armstrong JJ, Wasiuta T, Kiatos E, Malvankar-Mehta M, Hutnik CML. The effects of phacoemulsification on intraocular pressure and topical medication use in patients with glaucoma: a systematic review and meta-analysis of 3-year data. J Glaucoma. 2017;26:511-22. 